\title{
Silencing microRNA rescues the heart
}

Heart failure remains a main cause of morbidity and mortality, and anticipated breakthroughs in treatment in recent years have not materialized. Writing in Nature, Thum and colleagues show that antagonism of a single microRNA (miRNA) may rectify deleterious signalling pathways in heart failure, unveiling a potential strategy for disease prevention and treatment.

Heart failure is a progressive disorder that is initiated by myocardial injury and is characterized by fibrosis, hypertrophy and cardiac dysfunction. Previous studies have revealed miRNAs to be involved in the regulation of gene expression in the diseased heart, but so far their precise pathophysiological role and their validity as therapeutic targets have not been confirmed.

To explore this, Thum and colleagues first set out to identify

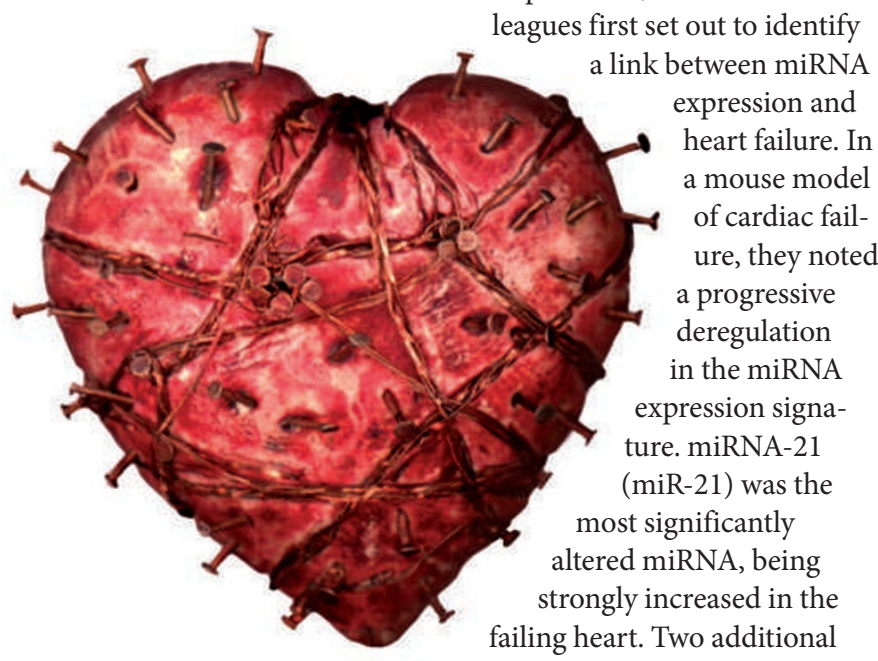

mouse models confirmed this observation, which importantly also held true in failing human myocardium.

The authors next investigated a potential role for increased miR-21 expression in heart failure. In vitro, a synthetic miR-21 antagonist increased the percentage of apoptotic fibroblasts, whereas miR-21 overexpression decreased it. These observations are of great importance, as cardiac fibroblasts have a central role in mediating the interstitial fibrosis and cardiac remodelling of the failing heart.

Mechanistic studies showed that the activation of the mitogenactivated protein kinase (MAPK) extracellular signal-regulated kinase (ERK) was probably mediating the miR-21-induced protection from apoptosis, as this effect was blocked by the ERK inhibitor PD98059. Moreover, a potent inhibitor of the RAS-MAPK/ERK kinase-ERK signalling pathway, sprouty homologue 1 (SPRY1), was shown to be involved.

Transfection of rat primary cardiac fibroblasts with miR-21 precursors strongly repressed SPRY1 expression, thereby increasing ERK activity and cell survival. By contrast, co-transfection with miR-21 precursors and a miR-21-resistant Spry1 mutant completely abolished any anti-apoptotic effect of miR-21. Spry1 repression had a similar effect in mice. Finally, consistent with these observations, SPRY1 expression was repressed and ERK was activated in failing human myocardium.

Subsequent studies explored the potential of using miR-21 as a therapeutic target. Mice subjected to experimental heart failure transverse aortic constriction (TAC) - were injected with an antisense oligonucleotide specific for miR-21 (antagomir-21) for 3 consecutive days. This repressed cardiac miR-21 for up to 3 weeks and completely reversed changes in SPRY1 expression and ERK activation. Moreover, the increased interstitial fibrosis, cardiomyocyte size, heart weight and cardiac dysfunction that were observed 3 weeks after TAC in control mice were significantly attenuated. Antagomir-21 exerted analogous beneficial effects when mice were treated 3 weeks after TAC was carried out, indicating the therapeutic potential of silencing miR-21 in more established heart disease.

Overall, these findings identify a primary role for miR-21 and cardiac fibroblast activity in heart failure and provide further support for the development of miRNA therapeutics.

Sarah Crunkhorn

ORIGINAL RESEARCH PAPER Thum, T. et al. MicroRNA-21 contributes to myocardial disease by stimulating MAP kinase signalling in fibroblasts. Nature 456, 980-984 (2008) FURTHER READING Kaye, D. \& Krum H. Drug discovery for heart failure: a new era or the end of the pipeline? Nature Rev. Drug Discov. 6 , 127-139 (2007) 